\title{
Uso de crioprecipitado em cão com hemorragia aguda: relato de caso*
}

\section{The use of cryoprecipitate in a dog with acute hemorrhage: case report}

\author{
Ludmila Rodrigues Moroz, ${ }^{* *}$ Claudia Medina de Oliveira, ${ }^{* * *}$ Fabio Hosoi Rezende, ${ }^{* * *}$ Denise Tabacchi Fantoni ${ }^{* * * *}$
}

\begin{abstract}
Resumo
Relata-se o caso do uso de crioprecipitado em um paciente canino, Yorkshire Terrier, que apresentou sangramento agudo intenso durante procedimento de artroplastia. No momento do distúrbio hemorrágico o paciente possuía valores de tromboplastina parcial ativada (TTPA) acima de 240 segundos, e de tempo de protrombina (TP) de 14,9 segundos. Optou-se pelo uso de hemocomponentes plasmáticos a fim de corrigir os distúrbios hemorrágicos, e uma vez que o paciente possuía peso reduzido $(4,8 \mathrm{~kg})$, foi decidido pela transfusão de uma unidade de crioprecipitado $(20 \mathrm{~mL})$, de forma rápida (20 minutos), ainda no trans-operatório. Os valores de TTPA e TP pós-transfusionais foram de 45,5 e 10,2 segundos, respectivamente, com rápido controle do sangramento. No retorno após 13 dias os valores de TTPA e TP estavam dentro dos valores de normalidade para a espécie. Conclui-se que o crioprecipitado ofereceu os fatores de coagulação necessários para correção do distúrbio hemorrágico, controlando o sangramento e melhorando os valores de TTPA e TP. Por conter alta concentração de fatores de coagulação e volume reduzido, o crioprecipitado pode ser uma boa escolha para pacientes com coagulopatias secundárias, especialmente em animais de pequeno porte. A eficácia na terapia transfusional direcionada com o uso racional do melhor hemocomponente para o caso ilustra a necessidade de conhecimento dos diferentes hemocomponentes e estudo individual dos casos.
\end{abstract}

Palavras-chave: hemostasia, distúrbios de coagulação, hemoterapia, medicina transfusional veterinária, hemocomponentes.

\begin{abstract}
It is reported the case of the use of cryoprecipitate in a canine patient, Yorkshire Terrier, who presented acute heavy bleeding disorder during arthroplasty procedure. At the time of bleeding disorder the patient had values of activated partial thromboplastin time (aPTT) above 240 seconds, and prothrombin time (PT) of 14.9 seconds. It was opted for the use of plasma components to correct the bleeding disorders, and since the patient had low weight $(4.8 \mathrm{~kg})$, it was decided by the transfusion of one unit of cryoprecipitate $(20 \mathrm{~mL})$, quickly (20 minutes), even during surgery. The aPTT and PT post-transfusion values were 45.5 and 10.2 seconds respectively, with rapid control of bleeding. On return after 13 days the values of APTT and PT were within the normal range for the species. Because it contains high concentration of clotting factors and reduced volume, cryoprecipitate may be a good choice for patients with secondary bleeding disorders, especially in small animals. The effectiveness in transfusion therapy directed to the rational use of blood components illustrates the need for knowledge of the different blood components and individual study of each case.
\end{abstract}

Keywords: hemostasis, bleeding disorders, hemotherapy, veterinary transfusion medicine, blood components.

\section{Introdução}

A utilização de componentes sanguíneos refere-se à administração de partes específicas do sangue necessárias pelo paciente, direcionando o tratamento de forma individual. Esta forma de trabalhar o sangue permite conservar estoques sanguíneos, reduzir o volume transfundido e diminuir os riscos de reações transfusionais, além de permitir que cada doação possa beneficiar mais de um paciente, de forma mais específica (AABB, 1998; Pereira e Reichman, 2002; Davidow, 2013; Moroz e Vieira, 2015). No caso de distúrbios hemorrágicos, a administração de sangue total pode não conferir a quantidade adequada de fatores de coagulação, podendo ainda causar hipervolemia ou policitemia em animais que não estejam anêmicos.
O crioprecipitado é proveniente do reprocessamento de uma bolsa padrão (220-250mL) de plasma fresco congelado (PFC) por meio de descongelamento lento em baixas temperaturas (em torno de $4^{\circ} \mathrm{C} \pm 2$ ). Esse processo de descongelar lentamente em baixa temperatura fará a precipitação de proteínas hemostáticas, e ao realizar nova centrifugação em alta rotação, haverá a sedimentação destes fatores. O precipitado é separado do sobrenadante de forma asséptica e em sistema fechado, procedimento possível ao se utilizar bolsas de coleta múltiplas. O precipitado é novamente congelado (agora classificado como crioprecipitado) e mantido em temperaturas abaixo de $-18^{\circ} \mathrm{C}$ tendo validade de 12 meses a partir da data da colheita. O volume do crioprecipitado gira em torno de 15 a $25 \mathrm{~mL}$ de plasma. O plasma sobrenadante restante pode ser

*Recebido em 16 de setembro de 2014 e aceito em 1 de novembro de 2015.

**Universidade Federal da Bahia, Laboratório de Análises Clínicas do Hospital Veterinário.

***Médico Veterinário autônomo, Sào Paulo-SP, Brasil.

${ }^{* * * *}$ Programa de Clínica Cirúrgica (VCl) da Faculdade de Medicina Veterinária e Zootecnia da Universidade de São Paulo (FMVZ-USP) Autor para correspondência: Ludmila Rodrigues Moroz. ludymoroz@gmail.com 
congelado, sendo classificado como crioplasma pobre, sendo que este possui albumina e imunoglobulinas, com validade de 5 anos a partir da data da colheita (Feldman e Sink, 2007; Moroz e Vieira, 2015).

O crioprecipitado é um hemocomponente rico em fatores de coagulação, possuindo alta concentração de fatores I (fibrinogênio), VIII (fator anti-hemofílico), XIII (fator estabilizador de fibrina) e fator de von Willebrand (FvW) (AABB, 1998; Stokol e Parry, 1998; Davidow, 2013). Ao se comparar com o PFC possui cerca de 80 vezes mais fator VIII, $250 \mathrm{mg}$ de fibrinogênio e cerca de 40 a $70 \%$ do FvW da bolsa inicial (AABB, 1998). Isto confere ao crioprecipitado uma efetividade maior no que se refere à correção de distúrbios hemorrágicos.

As principais indicações de uso de crioprecipitado são para correção de distúrbios hemostáticos ocasionados por hemofila A, doença de von Willebrand (DvW) e hipofibrinogenemia (como a que pode ocorrer na coagulação intravascular disseminada, hemodiluição maciça ou falha na síntese) (Stokol e Parry, 1995; AABB, 1998; Stokol et al., 1997; Ketchum et al., 2006)

Este artigo descreve o uso de crioprecipitado em cão que desenvolveu distúrbio hemorrágico durante procedimento cirúrgico, discutindo as mudanças na clínica do paciente e variação pré e pós-transfusional dos tempos de coagulação.

\section{Relato do caso}

Um cão da raça Yorkshire Terrier, com seis anos, pesando $4,8 \mathrm{~kg}$, foi encaminhado ao setor de cirurgia para ser submetido a um procedimento de artroplastia e estabilização de joelho esquerdo devido a luxação de patela medial grau III e ruptura de ligamento cruzado cranial. Seus exames pré-anestésicos, realizados sete dias antes, estavam dentro dos padrões de normalidade, com exceção da ureia que se apresentava ligeiramente elevada.

O cão recebeu como medicação préanestésica acepromazina $(0,05 \mathrm{mg} / \mathrm{kg})$ e meperidina $(2 \mathrm{mg} / \mathrm{kg}$ ) pela via intramuscular. A indução foi realizada com propofol $(5 \mathrm{mg} / \mathrm{kg}$ IV) e a manutenção anestésica realizada com isofluorano em $100 \%$ de $\mathrm{O}_{2}$. Como técnica de anestesia regional administrou-se fentanil $(2 \mu \mathrm{g} / \mathrm{kg})$, lidocaína $(4 \mathrm{mg} / \mathrm{kg})$ e morfina $(0,1$ $\mathrm{mg} / \mathrm{kg}$ ) pela via epidural no espaço lombosacro. Após administração de bloqueador neuromuscular (rocurônio 0,6 mg/kg) deu-se início a ventilação mecânica. A fluidoterapia foi mantida com Ringer lactato na taxa de $5 \mathrm{~mL} / \mathrm{kg} /$ hora e o animal foi monitorado por meio de eletrocardiografia, capnografia e pressão arterial invasiva obtida em artéria podal.

Após cerca de duas horas do início da artroplastia, o cirurgião atentou ao sangramento excessivo apresentado pelo animal, dificultando manipulação e suturas. A pressão arterial, valores de dióxido de carbono em ar expirado assim como $\mathrm{pH}$ sanguíneos estavam dentro dos padrões de normalidade.

Foi encaminhado sangue venoso para avaliação do tempo de tromboplastina parcial ativada (TTPA) e do tempo de protrombina (TP) em laboratório interno. O resultado do TTPA foi acima de 240 segundos (repetido em quadruplicata), e TP 14,9 (média da duplicata). A fim de determinar melhor a deficiência apresentada pelo paciente, foi realizado em seguida o teste de TTPA 1:1 (acrescentar uma parte de plasma de animal normal foi adicionada uma parte de plasma do paciente) com resultado de 47,2 segundos.

Frente ao sangramento agudo grave e aos resultados obtidos em laboratório, optou-se pela reposição de fatores de coagulação. Dentre as duas opções possíveis (plasma fresco congelado ou crioprecipitado) optou-se pelo crioprecipitado, uma unidade (20 $\mathrm{mL}$ ) administrada no período transoperatório. O volume globular (VG) foi de $22 \%$ ao final do procedimento anestésico, não sendo realizada transfusão de concentrado de hemácias.

Ao final da transfusão de crioprecipitado o sangramento estava controlado e os exames de TTPA e TP foram repetidos, sendo os resultados 45,5 e 10,2 segundos, respectivamente. O paciente foi monitorado por seis horas, e levado para cuidados domiciliares uma vez que os parâmetros fisiológicos não indicavam risco de piora clínica. Após 12 horas, o cão retornou para avaliação, estando alerta, mas com hematoma em região inguinal e ventral de abdome e tórax (Figura 1), e apresentando VG de $27 \%$.

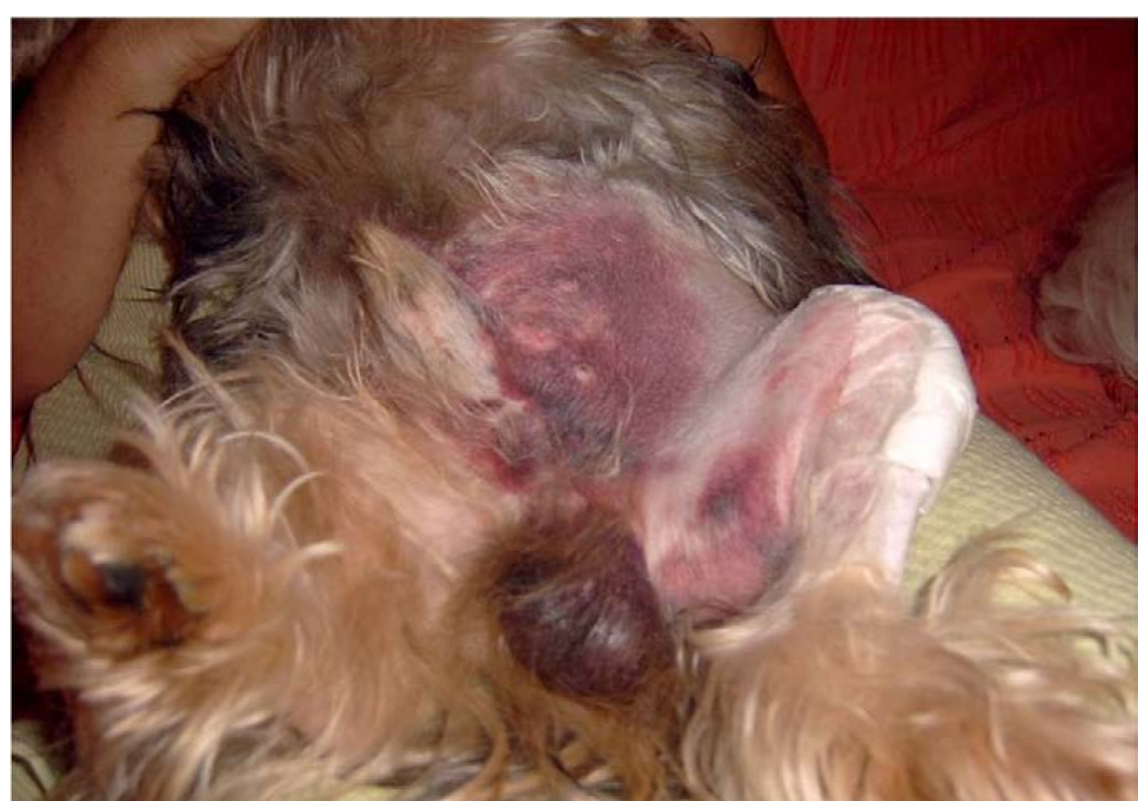

Figura 1: Cão, raça Yorkshire, 6 anos com hematoma em região abdominal ventral e bolsa escrotal, face medial e caudal de membro pélvico esquerdo, um dia após o procedimento cirúrgico de artroplastia.

Após 13 dias, o animal retornou para nova reavaliação estando em bom estado geral, alerta, sendo que o hematoma se encontrava quase ausente. Os resultados dos exames estão apresentados na Tabela 1. 
Tabela 1: Valores de resultados de exames realizados em períodos pré, trans e póscirúrgico de paciente canino, raça Yorkshire, 6 anos, submetido a cirurgia de artroplastia

\begin{tabular}{lccccc}
\hline & $\begin{array}{c}\text { 1 semana } \\
\text { pré-cirúrgico }\end{array}$ & $\begin{array}{c}\text { TRANS } \\
\text { Pré-CRIO }\end{array}$ & $\begin{array}{c}\text { TRANS } \\
\text { Pós-CRIO }\end{array}$ & $\begin{array}{c}\text { 13 dias pós } \\
\text { cirúrgico }\end{array}$ & $\begin{array}{c}\text { Referências de } \\
\text { normalidade }\end{array}$ \\
\hline VG $(\%)$ & 57 & 22 & & 49,5 & $37-55$ \\
Plaquetas $(/ \mu \mathrm{L})$ & 336.000 & & 554.000 & $200.000-500.000$ \\
Uréia $(\mathrm{mg} / \mathrm{dL})$ & 77,7 & & 63,8 & $7-28$ \\
Creatinina $(\mathrm{mg} / \mathrm{dL})$ & 1,2 & & 1,2 & $0,9-1,7$ \\
FA (U/L) & 71 & & 17,9 & $35-280$ \\
ALT (U/L) & 31,5 & & 25,7 & $10-120$ \\
PT (g/dL) & 6,4 & & 5,9 & $5,4-7,4$ \\
Albumina (g/dL) & 3,5 & & & 3,0 & $2,7-4,5$ \\
TTPA (segundos) & & $>240$ & 45 & 15,05 & $15,1 \pm 1,6$ \\
TTPA (1:1) & & 47,2 & & & \\
TP (segundos) & & 14,9 & 10,2 & 7,5 & $6,87 \pm 1,4$ \\
\hline
\end{tabular}

Referência (hematologia e bioquímica sérica) - THRALL, 2006. Referência para TTPAe TP - TAKAHIRA, 2015.

Abreviaturas: VG - volume globular; FA - fosfatase alcalina; ALT - alanina aminatransferase; PT proteínas totais séricas; TTPA - tempo de tromboplastina parcial ativada; TP - tempo de protrombina; CRIO - crioprecipitado, TRANS - transcirúrgico.

\section{Discussão}

Distúrbios de coagulação hemorrágicos ocorrem em decorrência da falha da hemostasia primária (plaquetas e endotélio vascular), secundária (formação de fibrina) ou ambas. Reconhecer clinicamente e diagnosticar rapidamente qual via da hemostasia está prejudicada é de fundamental importância para correção imediata e de forma específica. Há sinais clínicos capazes de guiar o clínico a iniciar uma abordagem inicial, além de ferramentas laboratoriais para confirmação da desordem na coagulação. Distúrbios na hemostasia primária costumam vir acompanhados de sangramentos superficiais e de mucosas (petéquias, equimoses, epistaxe, hematoemese, hematoquesia, hematúria), enquanto que distúrbios hemostáticos secundários cursam com sangramentos profundos, formação de hematomas e sangramentos em cavidades (hemoperitôneo, hemartroses) (Barr e Mcmichael, 2012; Herring e Mcmichael, 2012).

No caso apresentado, ocorreu uma coagulopatia adquirida, pois os exames laboratoriais pré e pós-operatórios de 7 e 13 dias, respectivamente, não demonstraram alterações, e as manifestações clínicas iniciaram-se 2 horas do início do procedimento cirúrgico. Outro ponto que faz afastar uma possível coagulopatia hereditária é pelo fato de o animal nunca ter apresentado sinal de disfunção na coagulação. Não havia relatos pregressos de sangramentos de qualquer ordem, sendo que o cão já fora submetido a tratamento periodontal e extração de dentes decíduos sem qualquer intercorrência.

Entre as causas prováveis, o emprego de solução heparinizada de forma inadvertida para manutenção do cateter para mensuração da pressão invasiva pode ser apontada como causa desencadeante. No total, o animal recebeu $750 \mathrm{Ul}$ de heparina via intravenosa durante todo o procedimento, sendo o equivalente a 156,25 Ul/kg. Apesar de estar dentro dos valores indicados para a terapia anticoagulante de cães, os limites mínimos e máximos recomendados são muito discrepantes, podendo variar entre 75 a $1000 \mathrm{UI} /$ $\mathrm{Kg}$, de acordo com a indicação clínica para o paciente (Diquelou et al., 2005; Takahira, 2015).

Um estudo avaliando o efeito da heparina na formação de trombina e determinação de TTPA em cães hígidos observou que aumentos de TTPA acima de 150 segundos indicam uma concentração plasmática de heparina acima de $1,5 \mathrm{Ul} / \mathrm{mL}$. Neste mesmo estudo a adição in vitro de $0,4 \mathrm{U} / \mathrm{mL}$ de heparina foi suficiente para bloquear completamente a geração de trombina (Allegret et al., 2011). Outro estudo apontou que concentrações de $0,7 \mathrm{UI} / \mathrm{L}$ são capazes de aumentar em mais de duas vezes os valores de normalidade de TTPA para a espécie canina (Mischke e Jacobs, 2001). Desta forma, pode-se supor que o paciente do caso apresentado estivesse com uma concentração plasmática de heparina acima do recomendado para controle trombótico. Ainda que os estudos com uso de heparina em cães indiquem que os aumentos nos valores de TTPA não foram suficientes para causar diátese hemorrágica, todos foram realizados em pacientes hígidos que não foram submetidos a procedimentos anestésicos ou cirúrgicos (Mischke e Jacobs, 2001; Diquelou et al., 2005; Allegret et al., 2011; Mischke et al., 2001). Assim sendo, o paciente em questão pode ter desenvolvido a hemorragia pela combinação de mais fatores: presença de inibidor hemostático, lesão tecidual ativa com perda importante de sangue, hemodiluição pela fluidoterapia de reposição e hipotermia ocasionada pela anestesia (Stainsby et al., 2000; Dirkmann et al., 2008).

Partindo do princípio que o VG pré-cirúrgico era de $57 \%$ e antes da transfusão do crioprecipitado chegou a $22 \%$, pode-se supor que o animal tenha perdido, pelo menos metade de seu volume sanguíneo, mantendo a volemia com uso de soluções intravenosas (Ketchum et al., 2006). O uso da acepromazina como medicação pré-anestésica poderia ter influenciado na queda do VG no período transcirúrgico em decorrência de movimentação eritroide para o baço. Contudo, a manutenção deste baixo valor após 12 horas da transfusão do crioprecipitado (27\%) indicou perda sanguínea real, e não sequestro esplênico. Desta forma, destaca-se que o animal apresentou perda de sangue com manutenção volêmica por meio de reposição com cristaloides.

O teste de correção com plasma normal e nova determinação de TTPA indicou deficiência na via intrínseca dos fatores VIII e XI (Carvalho, 2004). Desta forma, o crioprecipitado seria um bom hemocomponente por possuir maior concentração de fator VIII, além de fator de von Willebrand, fibrinogênio e de fator XVIII quando comparado ao PFC, melhorando a agregação plaquetária e estabilização da fibrina formada (Ketchum et al., 
2006; Barr e Mcmichael, 2012; Davidow, 2013). Apesar de não ter sido realizado o teste de correção 1:1 para TP por falta de plasma para teste, foi possível observar que o crioprecipitado não teve o mesmo impacto em TTPA e TP. Enquanto a redução do valor de TTPA foi de, pelo menos, $81,25 \%$, a redução de TP foi de $31,54 \%$. A heparina é um inibidor da coagulação com alto impacto no TTPA e baixo efeito no TP, portanto, pode-se supor que o efeito no TP foi ocasionado pela diluição ocorrida durante a anestesia.

Outro fator preponderante para a escolha do crioprecipitado em vez do PFC foi o volume, uma vez que o paciente era de pequeno porte $(4,8 \mathrm{Kg})$, estava normotenso e necessitava de uma correção imediata dos fatores de coagulação a fim de estancar o sangramento. Uma vez que o crioprecipitado possui cerca de $4 \%$ do volume do PFC este pode ser infundido de forma rápida (20 minutos). O mesmo não poderia ser realizado com PFC, podendo se refletir em maior tempo para a correção da coagulopatia, de 1 a 2 horas até o final da transfusão. Enquanto a dose de crioprecipitado seria de uma unidade (20 $\mathrm{ml}$ totais) a cada $10 \mathrm{~kg}$, a de PFC seria de, no mínimo, $96 \mathrm{~mL}$ transfundidos (dose de $20 \mathrm{~mL} / \mathrm{kg}$ ). Esse volume quatro vezes menor que poderia ser aplicado num período rápido em um animal normovolêmico foi o principal critério que resultou na escolha do crioprecipitado.

Esta opção ressalta a indicação de hemocomponentes como terapia direcionada para tratar o foco do distúrbio, ofertando produtos específicos, reduzindo riscos de reações transfusionais e otimizando cada coleta de sangue. Ademais, ao se abordar o risco transfusional, o crioprecipitado possui menor risco de reações transfusionais, principalmente a reação alérgica (hipersensibilidade tipo I), quando comparado com o uso de PFC (Stokol e Parry, 1998).

O crioprecipitado ainda é pouco utilizado na rotina veterinária, talvez pela baixa oferta, mas também pela baixa procura dos médicos-veterinários. Este pode ser utilizado com eficiência para repor fatores de coagulação de pacientes com hemofilia A, Doença de von Willebrand, complicações obstétricas e outras alterações que cursem com o consumo de fibrinogênio (Stokol e Parry, 1998; Ketchum et al., 2006).

Apesar do paciente ter apresentado uma redução severa no VG, caindo de $57 \%$ na semana anterior à cirurgia para $22 \%$ antes da transfusão de crioprecipitado, não houve mudanças nos parâmetros vitais (frequência cardíaca, respiratória, débito

\section{Referências}

AABB. Terapêutica transfusional. In: BANKS, A. A. OF B. (Ed.). Manual para médicos. 4. ed. [s.I.] AABB, 1998. p. 28-31.

ALLEGRET, V.; DUNN, M.; BÉDARD, C. Monitoring unfractionated heparin therapy in dogs by measuring thrombin generation. Veterinary clinical pathology / American Society for Veterinary Clinical Pathology, v. 40, n. 1, p. 24-31, mar. 2011.

BARR, J. W.; MCMICHAEL, M. Inherited disorders of hemostasis in dogs and cats. Topics in companion animal medicine, v. $27, \mathrm{n}$. 2, p. 53-58, maio 2012.

CARVALHO, A. C. A. Hemostasia e Trombose. In: SCHIFFMAN, F. J. (Ed.). . Fisiopatologia Hematológica. 1. ed. Santos: Editora Santos, 2004. p. 161-243. urinário ou pressão arterial) que indicassem redução da oferta de oxigênio aos tecidos, tampouco hipoxemia ou acidose (avaliado por meio de hemogasometria seriada). Essa manutenção da estabilidade hemodinâmica é reflexo da manutenção de um estado de normovolemia, apesar da hemorragia, e a decisão de evitar o uso de hemocomponentes eritrocitários foi baseada em estudos anteriores de hemodiluição normovolêmica aguda (HNA) (Van der Linden et al., 1998; Fantoni et al., 2005; Jamnicki et al., 2003). Entretanto, deve-se ressaltar que esta abordagem restritiva só foi possível mediante controle rígido dos parâmetros vitais e de equipe treinada na técnica de HNA, mantendo uma hemodiluição normovolêmica permissiva, e manutenção de uma bolsa previamente testada e reservada caso houvesse desestabilização clínica (Madjdpour e Spahn, 2005; Madjdpour et al., 2006).

Uma das formas de manter a normovolemia do paciente consistiu na infusão de fluidos cristaloides em volume suficiente para manter pressão arterial estável. Desta forma, houve uma diluição sanguínea que foi revertida após redistribuição hídrica entre os compartimentos sanguíneos (diurese e expiração) sendo que este efeito foi observado no aumento de VG de 22 para $27 \%$ em cerca de 12 horas.

Uma vez que não houve alterações cardiovasculares que indicassem redução da oferta de oxigênio tecidual (especialmente a estabilidade da frequência cardíaca e respiratória dentro dos valores de normalidade canina) e o conhecimento do estado de hemodiluição (com esperado aumento no valor do VG nos pós-operatório), ilustra o quanto uma terapia no uso de hemocomponentes de forma guiada e específica pode ser eficiente e trazer menos riscos ao paciente quando comparado ao uso de sangue total. Neste caso, menor risco de reações hemolíticas e sensibilização quando comparado ao uso de hemocomponentes eritrocitários e menor risco de sobrecarga circulatória ao optar por crioprecipitado em vez de PFC.

\section{Conclusão}

O uso do crioprecipitado mostrou-se eficiente em controlar o sangramento profuso no trans-cirúrgico, com rápida correção da hemorragia e melhora dos valores de TTPA e TP.

A eficácia na terapia transfusional direcionada com o uso racional do melhor hemocomponente para o caso, reduzindo riscos transfusionais, ilustra a necessidade de conhecimento dos diferentes hemocomponentes e estudo individual de cada caso.

DAVIDOW, B. Transfusion medicine in small animals. The Veterinary clinics of North America. Small animal practice, v. 43, n. 4, p. 735-756, jul. 2013.

DIQUÉLOU, A. et al. Pharmacokinetics and pharmacodynamics of a therapeutic dose of unfractionated heparin $(200 \mathrm{U} / \mathrm{kg})$ administered subcutaneously or intravenously to healthy dogs. Veterinary clinical pathology, v. 34, n. 3, p. 237-42, set. 2005.

DIRKMANN, D. et al. Hypothermia and acidosis synergistically impair coagulation in human whole blood. Anesthesia and Analgesia, v. 106, n. 6, p. 1627-32, jun. 2008.

FANTONI, D. T. et al. A comparative evaluation of inhaled halothane, isoflurane, and sevoflurane during acute normovolemic hemodilution in dogs. Anesthesia and analgesia, v. 100, n. 4, p. 1014-1019, abr. 2005. 
FELDMAN, B. F.; SINK, C. A. Hemoterapia para o clínico de pequenos animais. 1. ed. São Paulo: Hemoterapia para o clínico de pequenos animais, 2007.

HERRING, J.; MCMICHAEL, M. Diagnostic approach to small animal bleeding disorders. Topics in companion animal medicine, v. 27, n. 2, p. 73-80, maio 2012.

JAMNICKI, M. et al. Acute normovolemic hemodilution: physiology, limitations, and clinical use. Journal of Cardiothoracic and Vascular Anesthesia, v. 17, n. 6, p. 747-754, 2003.

KETCHUM, L.; HESS, J. R.; HIIPPALA, S. Indications for early fresh frozen plasma, cryoprecipitate, and platelet transfusion in trauma. The journal of trauma, v. 60, n. 6 Suppl, p. S51-58, jun. 2006.

MADJDPOUR, C.; HEINDL, V.; SPHAN, D. R. Risks, benefits, alternatives and indications of allogenic blood transfusions. Minerva anestesiologica, v. 72, p. 283-298, 2006.

MADJDPOUR, C.; SPAHN, D. R. Allogeneic red blood cell transfusions: efficacy, risks, alternatives and indications. British journal of anaesthesia, v. 95, n. 1, p. 33-42, jul. 2005.

MISCHKE, R. H.; SCHÜTTERT, C.; GREBE, S. I. Anticoagulant effects of repeated subcutaneous injections of high doses of unfractionated heparin in healthy dogs. American Journal Of Veterinary Research, v. 62, n. 12, p. 1887-1891, 2001.

MISCHKE, R.; JACOBS, C. The monitoring of heparin administration by screening tests in experimental dogs. Research in veterinary science, v. 70, n. 2, p. 101-108, abr. 2001.
MOROZ, L. R.; VIEIRA, J. Capítulo 209 - Transfusão sanguínea em cães. In: JERICÓ, M. M. (Ed). Tratado de medicina interna de cães e gatos. Roca:, 2015, v. 2; p. 1903-1931.

PEREIRA, P. M.; REICHMANN, P. Capítulo 19 - Fluidoterapia e transfusão sanguínea, Transfusão de sangue e seus derivados. In: ANDRADE, S. (Ed.). . Manual de Terapêutica Veterinária, 3. Ediçao, Editora Roca, 2008. p. 579-591.

STAINSBY, D.; MACLENNAN, S.; HAMILTON, P. J. Management of massive blood loss: a template guideline. British journal of anaesthesia, v. 85, n. 3, p. 487-91, set. 2000.

STOKOL, T. et al. Pharmacokinetics of von Willebrand factor and factor VIII in canine von Willebrand disease and haemophilia A. Research in Veterinary Science, v. 63, n. 1, p. 23-7, 1997.

STOKOL, T.; PARRY, B. W. Stability of von Willebrand factor and factor VIII in canine cryoprecipitate under various conditions of storage. Research in Veterinary Science, v. 59, n. 2, p. 152-5, set. 1995 .

STOKOL, T.; PARRY, B. W. Efficacy of fresh-frozen plasma and cryoprecipitate in dogs with von Willebrand's disease or hemophilia A. Journal of Veterinary Internal Medicine, v. 12, n. 2, p. 84-92, 1998.

TAKAHIRA, R. K. Capítulo 208 - Defeitos hemostáticos secundários. In: JERICÓ, M. M. (Ed). Tratado de medicina interna de cães e gatos. Roca:, 2015, volume 2; p. 1895-1902.

VAN DER LINDEN, P. et al. Critical haemoglobin concentration in anaesthetized dogs: comparison of two plasma substitutes. British journal of anaesthesia, v. 81, n. 4, p. 556-562, out. 1998. 